\title{
Repeated Bronchoscopy - Treatment of Severe Respiratory Failure in a Fire Victim
}

\author{
Ovidiu Rusalim Petris ${ }^{1}$, Cristina Bologa2*, Victorita Sorodoc², Catalina Lionte ${ }^{2}$ \\ ${ }^{1}$ Nursing Department, 2 ${ }^{\text {nd }}$ Internal Medicine Clinic, "Sf. Spiridon” Clinical County Emergency Hospital, “Gr. T. Popa" \\ University of Medicine, lasi, Romania \\ 2 Internal Medicine Department, $2^{\text {nd }}$ Internal Medicine Clinic, "Sf. Spiridon" Clinical County Emergency Hospital, "Gr. \\ T. Popa" University of Medicine, Iasi, Romania
}

\begin{abstract}
A case of respiratory failure in a domestic fire victim presenting with 1-3-degree skin burns on $10 \%$ of the total body surface, is reported. Forty-eight hours after admission to hospital, the patient developed severe respiratory failure that did not respond to mechanical ventilation. Severe obstruction of the airway had resulted from secretions and deposits of soot forming bronchial casts. The patient required repeated bronchoscopies to separate and remove the bronchial secretions and soot deposits. An emergency bronchial endoscopic exam was crucial in the patient's survival and management. The patient was discharged from the hospital after twenty-four days.
\end{abstract}

Keywords: smoke inhalation injury, burns, bronchial soot deposits, bronchoscopy, respiratory failure

Received: 3 March 2017 / Accepted: 30 July 2017

\section{INTRODUCTION}

Victims of domestic fire usually present with co-existing skin and respiratory lesions $[1,2]$. In many cases, upper airway lesions are often ignored. All good medical practice reports recommend that all fire victims should be systematically evaluated by bronchoscopy $[3,4]$.

Criteria for bronchoscopy assessment, to standardise the severity of respiratory lesions in domestic fire cases, have been published [5]. Bronchoscopy is considered to be the "gold standard" for early evaluation of upper airway injury in burns patients for predicting acute pulmonary lesions and treating these injuries $[3,6]$.

The current case reports the experience of using repeated bronchoscopies as a treatment tool in a case of severe respiratory failure due to bronchial obstruction by secretions and soot deposits in a burns patient. The patient was a victim of a domestic closed space fire who presented with 1-3-degree skin burns on $10 \%$ of the total body surface. Forty-eight hours from admission, he developed severe respiratory failure, requiring repeated bronchial lavages to remove secretions and soot deposits.

\section{CASE REPORT}

A 23-year-old man, victim of a closed space house fire, was first admitted to the Roman County Hospital, Romania and two days later was transferred to the Emergency County Clinic Hospital Iasi, Romania.

On admission, the physical examination revealed an overweight patient with a body mass index of 28.7 $\mathrm{kg} / \mathrm{m}^{2}$, with 1-3-degree skin burns on $10 \%$ of the total body surface involving areas of the face, left shoulder, arm and forearm, and both hands. He was conscious and haemodynamically stable. He had dyspnoea with polypnea ( 25 breaths/min), productive cough with yellow grey sputum, and bilateral rhonchi were detected on pulmonary auscultation. Chest X-rays revealed an area of pulmonary condensation in the superior lobe of the left lung, while the right lung area appeared radiologically normal. Arterial blood gases analysis while breathing room air showed $\mathrm{pO}_{2} 120 \mathrm{mmHg}, \mathrm{pCO}_{2} 60$ mmHg, $\mathrm{SaO}_{2}$ 94\%, $\mathrm{pH} 7.32$.

Laboratory tests showed marked leucocytosis $\left(34,100 / \mathrm{mm}^{3}\right)$ with neutrophilia, increased ASAT (73 U/l), CK (1868 U/l) and LDH (1161 U/l), increased lactate level (1.3 mmol/l), and glycaemia $-119 \mathrm{mg} / \mathrm{dl}$.

\footnotetext{
* Correspondence to: Cristina Bologa, Universitatea de Medicina si Farmacie "Gr.T.Popa" lasi. Str.Universitatii nr.16. 700115 lasi, Romania. E-mail: crisbologa@yahoo.com
} 
Bronchoscopy was delayed due to unforeseen technical difficulties.

Local treatment of the skin burns consisting of mechanical and chemical washing was commenced on the first day of admission along with broad spectrum antibiotic therapy with intravenous amoxicillin/clavulanate potassium $1000 \mathrm{mg} / 200 \mathrm{mg}$, every 8 hours for 14 days.

Forty-eight hours after admission, despite receiving supplemental oxygen via nasal cannula, the patient developed progressively severe dyspnoea and tachypnoea with a respiratory rate of $32 /$ minute, peripheral cyanosis and agitation, tachycardia (134/min), blood pressure $(40 / 80 \mathrm{mmHg}$ ) and decreased oxygen saturation (70\%). Arterial blood gas analysis showed $\mathrm{pO}_{2}, 41$ mmHg; $\mathrm{pCO}_{2}, 98 \mathrm{mmHg} ; \mathrm{pH}=7.18$, and $\mathrm{PaO}_{2} / \mathrm{FiO}_{2}=$ 102 , which were consistent with the diagnosis of respiratory failure. The patient was promptly intubated and mechanically ventilated. A bedside chest X-ray showed bilateral confluent multiple nodular lesions similar to those described in moderate acute respiratory distress syndrome (ARDS).

Despite repeated attempts to correct ventilation, only a limited improvement of arterial gases, was achieved. Mechanical ventilation using BiPAP mode with $\mathrm{FiO}_{2} \quad 100 \%$ which was progressively increased 5 to $7 \mathrm{~cm}$ water PEEP, improved the oxygen saturation to $86 \%$ and $\mathrm{pO}_{2}$ to $54 \mathrm{mmHg}$. The change of inspiratory pressure ( $\mathrm{Pi}$ ) from 25 to $35 \mathrm{~cm}$ water failed to improve the tidal volume, which remained at $350 \mathrm{ml}$. It was considered necessary to increase the respiratory rate to $16-18$ breaths/min to correct hypoventilation and to decrease $\mathrm{pCO}_{2}$.

At this point, it was suspected that an obstruction of the superior airway had occurred, and an emergency endoscopy was requested. This showed a grade 2 inhalation injury, numerous white-grey secretions and soot deposits which had formed bronchial casts causing severe airway obstruction (Fig. 1).

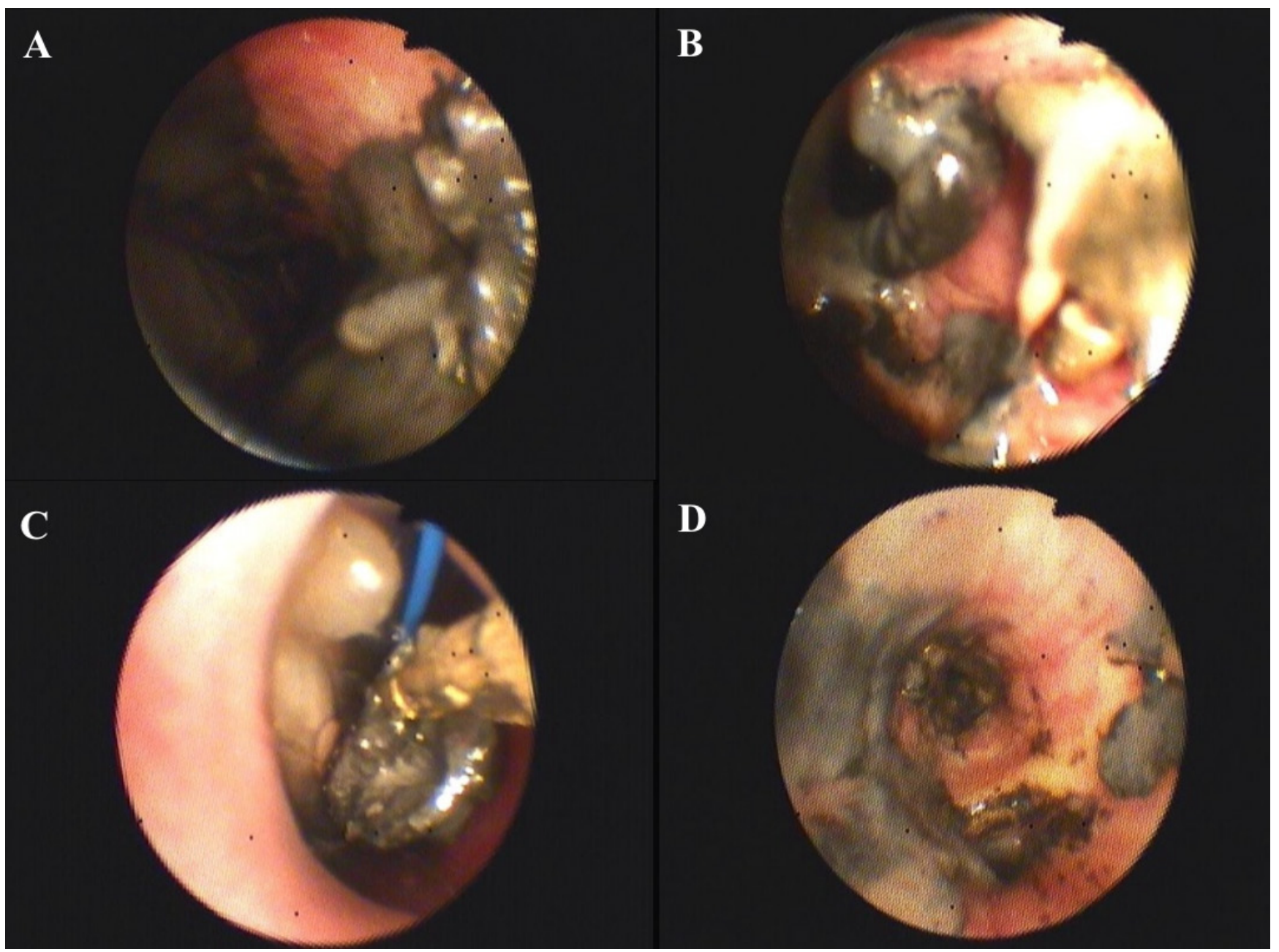

Fig. 1. Endoscopic appearance of the airways: A. Secretions and deposits of soot; B. Organized bronchial casts; C. Removal using bronchial forceps; D. Revealing a normal bronchial mucosa underneath the secretions. 
The removal of the bronchial casts required the use of biopsy forceps to detach the pseudo membranes and when completed, revealed a normal bronchial mucosa underneath. The respiratory status of the patient, as well as the parameters of arterial blood gases, improved after daily bronchial endoscopic lavages. Bronchoscopy was necessary for eight days to clean all bronchial secretions and soot deposits efficiently. Extubation was possible ten days after the development of the severe respiratory failure.

The serial direct microscopic exam of the samples collected daily revealed an inflammatory reaction, with neutrophils and fibrin. No bacterial growths were obtained from repeated culture of broncho alveolar specimens.

The skin burns and lesions of the upper airway responded favourably to supportive therapy, broad spectrum antibiotherapy and analgesia with remifentanil $0.075-1 \mathrm{mcg} / \mathrm{kg} / \mathrm{min}$, continuous infusion for 8 days.

The bronchoscopy performed just before discharge revealed no secretions, no luminal obstructions and no fistulas. days.

The patient was released from the hospital after 24

\section{Discussion}

Severe airways obstruction due to secretions and deposits of soot organised as bronchial casts was treated by repeated bronchial endoscopic examination. This proved to be an effective therapeutic method for removing bronchial soot casts, resulting in improved ventilator function and patient condition.

As reported in the literature on domestic fire victims with inhalational smoke injuries, upper airway lesions are often ignored. Pulmonary lesions usually cause problems which commence a few days following the fire and lead to worsening of the patient's vital prognosis. Serial bronchoscopies with lavage have been reported to be useful for clearing of mucus plugs. [1, 2, 5, 7-9].

There are contradictory opinions regarding serial fibre-optic bronchoscopies in patients with smoke inhalational injuries, because of the lack of prospective or interventional studies. [10] However, a study performed on 624 patients with smoke inhalational injuries showed a trend toward shorter hospitalisation in patients who underwent more than one bronchoscopy procedure [11].
Although there are multiple causes, in addition to smoke inhalation, which contribute to lung injury, such as systemic inflammation in response to burns, ventilator-induced injury or pulmonary infections [1], only smoke inhalation and systemic inflammation were considered to be responsible for the bronchial lesions.

Scoring systems have been formulated to identify patients with a poor prognosis, The Abbreviated Injury Score grading scale is one of them and is correlated with increased mortality in burns victims [12].

In the current case, bronchoscopy revealed a moderate inhalation injury. The patient developed a severe respiratory failure that could not be treated only by mechanical ventilation, and repeated bronchoscopies were required to separate and remove bronchial secretions and soot deposits before their organisation in bronchial casts.

There are two types of respiratory injuries that appear in domestic fire victims, injuries related to smoke inhalation and injuries related to the heat generated by a fire $[1,9]$. Patients with respiratory injuries have a poor prognosis and a mortality rate of up to $20 \%$ [1]. When there are associated pulmonary infections, the mortality rate can reach $40 \%[1,8]$.

The negative bronchial cultures obtained in the present case were probably accounted for by the broad spectrum antibiotic therapy initiated on admission.

Following the experience of using multiple bronchoscopies in this reported case, a bronchial endoscopy investigation protocol was implemented for all domestic fire victims admitted to the Burns Department of Iasi Clinical Emergency County Hospital. From January 2004 to December 2013, a total of 1759 cases of burnt patients were admitted to the Burns Department and flexible bronchoscopy was performed on all admitted patients. In most cases, mild to moderately severe lesions, graded 5 and 8 on the Jones' system of grading, were detected [5].

\section{- CONCLUSIONS}

Daily flexible bronchoscopies and airway washings for the treatment of respiratory failure from bronchial plugs secondary to lung injury after exposure to domestic fires lesions contributed to patient progress, which was not responding to treatment with mechanical ventilation alone. 


\section{CONFLICT OF INTEREST}

\section{None to declare}

\section{REFERENCES}

1. Dries DJ, Endorf FW. Inhalation injury: epidemiology, pathology, treatment strategies. Scand J Trauma Resusc Emerg Med. 2013;21:31.

2. Hassan Z, Wong JK, Bush J, Bayat A, Dunn KW. Assessing the severity of inhalation injuries in adults. Burns. 2010;36:212-6.

3. Bai C, Huang HD, YaoXP etal. Application offlexible bronchoscopy in inhalation lung injury. Diagn Pathol. 2013;8:174.

4. Mlcak RP, Suman OE, Herndon DN. Respiratory management of inhalation injury. Burns. 2007;33:2-13.

5. Jones SW, Zhou H, Ortiz-Pujols SM et al. Bronchoscopy-Derived Correlates of Lung Injury following Inhalational Injuries: A Prospective Observational Study. Plos One. 2013;8:e64250.

6. Marek K, Piotr W, Stanislaw S et al. Fiberoptic bronchoscopy in routine clinical practice in confirming the diagnosis and treatment of inhalation burns. Burns. 2007;33:554-60.

7. Traber DL, Hawkins HK, Enkhbaatar P et al. The role of the bronchial circulation in the acute lung injury resulting from burn and smoke inhalation. Pulm Pharmacol Ther. 2007;20:163-6.

8. Enkhbaatar P, Traber DL. Pathophysiology of acute lung injury in combined burn and smoke inhalation injury. Clin Sci. 2004;107:137-43.

9. Lee AS, Mellins RB. Lung injury from smoke inhalation. Paediatr Respir Rev. 2006;7:123-8.

10. Amin M, Shaarawy H, Gad El-Rab E. Role of fiberoptic bronchoscopy in management of smoke inhalation lung injury. Egyptian Journal of Chest Diseases and Tuberculosis. 2015;64:733-7.

11. Carr JA, Phillips BD, Bowling WM. The utility of bronchoscopy after inhalation injury complicated by pneumonia in burn patients: Results from the national burn repository. J Burn Care Res.2009;30:967-74.

12. Walker PF, Buehner MF, Wood LA. et al. Diagnosis and management of inhalation injury: an updated review. Crit Care. 2015;19:351. 